\title{
Acknowledgment of Reviewers, 2014
}

The success of Phytopathology depends on the quality of manuscripts submitted by authors and on the care and competence with which they are reviewed. It is the policy of the Editorial Board to solicit reviews for manuscripts from specialists who are most qualified to review them. In addition to members of the Editorial Board, the individuals listed below provide constructive critical reviews of one or more manuscripts during the past year. Their names are published here in grateful appreciation of their contribution to the journal and to the science of phytopathology. http://dx.doi.org/10.1094/PHYTO-105-1-0004

Adams, G. C., University of Nebraska, Lincoln, NE, U.S.A. Adaskaveg, J. E., University of California, Riverside, CA, U.S.A. Affeldt, K., University of Wisconsin, Madison, WI, U.S.A.

Alkan, N., Agricultural Research Organization, Volcani Center, BetDagan, Israel

Allen, T. W., Mississippi State University, Stoneville, MS, U.S.A. Almeida, R., University of California, Berkeley, CA, U.S.A. Alvarez, A., University of Hawaii, Honolulu, HI, U.S.A. Alvarez, E. C., Valle del Cauca, Colombia Anagnostakis, S., Connecticut Agricultural Experiment Station Aranda, M. A., CEBAS-CSIC, Murcia, Spain Armengol, J., Universidad Politécnica de Valencia, Valencia, Spain Asea, G.

Bag, S., University of California, Davis, CA, U.S.A.

Baishi, H., Nanjing, Jiangsu, China

Baker, J. C., USDA-ARS, Beltsville, MD, U.S.A.

Balci, Y., University of Maryland, College Park, MD, U.S.A.

Balota, M., Virginia Tech

Baltrus, D., University of Arizona, Tucson, AR, U.S.A.

Barash, I., U.S.A.

Bariana, H., University of Sydney, New South Wales, Australia

Bartz, F., Emory University, Atlanta, GA, U.S.A.

Baudoin, A., Virginia Tech, Blacksburg, VA, U.S.A.

Baysal, F. G., The Ohio State University, Wooster, OH, U.S.A.

Beaulieu, C., Université de Sherbrooke, Quebec, Canada

Beckerman, J., Purdue University, West Lafayette, IN, U.S.A.

Becktell, M., Colorado Mesa University, Grand Junction, CO, U.S.A.

Belanger, F., Rutgers University, East Brunswick, NJ, U.S.A.

Belanger, R., Laval University, Quebec, Canada

Belasque, J., University of Sao Paulo

Benson, M., North Carolina State University, Raleigh, NC, U.S.A.

Beresford, R. M., Plant \& Food Research, Auckland, New Zealand

Bertsch, C., Université de Haute Alsace, Strasbourg, France

Blok, V., The James Hutton Institute, Dundee, United Kingdom

Bock, C., USDA-ARS-USHRL, Ft. Pierce, U.S.A.

Bonman, J. M., USDA-ARS, Aberdeen, ID, U.S.A.

Bonnot, F., CIRAD, BIOS, Montpellier, France

Bové, J. M., INRA and University Victor Segalen Bordeaux, Villenave d'Ornon, France

Brandl, M. T., USDA-ARS, Albany, CA, U.S.A.

Brewer, M. T., University of Georgia, Athens, GA, U.S.A.

Broders, K., University of New Hampshire, Durham, NH, U.S.A.

Brown, J., John Innes Centre

Brown, J. K., University of Arizona, Tucson, AZ, U.S.A.

Brueggeman, R. S., North Dakota State University, Fargo, ND, U.S.A.

Burdman, S., The Hebrew University of Jerusalem, Rehovot, Israel

Burger, J., Stellenbosch University South Africa, Western Cape, South Africa

Burkey, K., USDA-ARS, Raleigh, NC, U.S.A.

Burr, T., Cornell University-NYSAES, Geneva, NY, U.S.A.

Candresse, T., INRA, UMR BFP, Villenave D'Ornon, France

Cao, A., Beijing, China

Cardwell, K. F., USDA, NIFA, District of Columbia, U.S.A.

Carisse, O., Agriculture and Agri-Food Canada, Quebec, Canada

Carter, A., Washington State University, Pullman, WA, U.S.A.

Cerkauskas, R., Agriculture \& Agri-Food Canada, Ontario Canada
Chang, C., University of Georgia, Griffin, GA, U.S.A.

Chastagner, G. A., Washington State University, Puyallup, WA, U.S.A.

Chatterjee, S., CDFD Hyderabad

Chaverri, P., University of Maryland, College Park, MD, U.S.A.

Chehri, K., Penang, Malaysia

Chellemi, D. O., USDA-ARS, USHRL, Fort Pierce, FL, U.S.A.

Chen, C.-Y., National Taiwan University, Taipei, Taiwan

Chen, G., Shanghai Jiao Tong University, Shanghai, China

Chen, J., USDA-ARS, Parlier, CA, U.S.A.

Chen, L.-F., University of California, Davis, CA, U.S.A.

Chen, W., Institute of Plant Protection, Beijing, China

Chen, W., Pullman, WA, U.S.A.

Chen, X., USDA-ARS and Washington State University, Pullman, WA, U.S.A.

Chernin, L., The Hebrew University of Jerusalem, Rehovot, Israel

Chilvers, M. I., Michigan State University, East Lansing, MI, U.S.A.

Cluzet, S., Université Bordeaux, Bordeaux, France

Coletta F., H. D., Cordeirópolis, Brazil

Cooke, D. E. L., Scottish Crop Research Institute, Dundee, United Kingdom

Cooke, D. E. L., The James Hutton Institute, Dundee, United

Kingdom

Cooley, D. R., University of Massachusetts, Amherst, MA, U.S.A.

Correa, F., RiceTec Inc., Alvin, TX, U.S.A.

Correll, J. C., University of Arkansas, Fayetteville, AR, U.S.A.

Costa, F., Fondazione Edmund Mach, San Michele all'Adige, Trento, Italy

Cotty, P., USDA-ARS SRRC, U.S.A.

Cox, K. D., Cornell University, Geneva, NY, U.S.A.

Crouch, J. A., USDA-ARS, Beltsville, MD, U.S.A.

Culbreath, A. K., University of Georgia, Tifton, GA, U.S.A.

Curtin, S., University of Minnesota, St. Paul, MN, U.S.A.

Cvrkovic, T., Institute for Plant Protection and Environment, Belgrade, Serbia

Dai, H., Qingdao Agricultural University, Qingdao City, China

Dalzell, J., Queen's University Belfast, Northern Ireland, United Kingdom

Danies, G., Cornell University, Ithaca, NY, U.S.A.

Datnoff, L. E., Louisiana State University, Baton Rouge, LA, U.S.A.

Davis, M., University of California, Davis, CA, U.S.A.

de la Fuente, L., Auburn University, Auburn, AL, U.S.A.

de Vallavieille-Pope, C., INRA, Thiverval-Grignon, France

De Wolf, E., Kansas State University, Manhattan, KS, U.S.A.

Dean, R., North Carolina State University, Raleigh, NC, U.S.A.

Degani, O., MIGAL-Galilee Research Institute, Kiryat-Shmona, Israel

Dickman, M., Institute for Plant Genomics and Biotechnology, College Station, TX, U.S.A.

Dixon, G., The University of Reading, United Kingdom do Amaral, A. M., EMBRAPA, SRI, Brasilia, DF, Brazil Dolezal, A., Monsanto, Waterman, IL, U.S.A.

Dong, H., Nanjing Agricultural University, Nanjing, China

Donofrio, N., University of Delaware, Newark, DE, U.S.A.

Duan, Y. P., USDA-ARS-USHRL, Fort Pierce, FL, U.S.A.

Dumenyo, K., Tennessee State University, Nashville, TN, U.S.A.

Dung, J. K. S., Oregon State University, Madras, OR, U.S.A. 
du Toit, L. J., Washington State University, Mount Vernon, WA, U.S.A.

Eastburn, D. M., University of Illinois, Urbana, IL, U.S.A.

Eastwell, K., Prosser, U.S.A.

Elad, Y., Volcani Center, Bet-Dagan, Israel

Elling, A. A., Washington State University, Pullman, WA, U.S.A.

Esker, P., Universidad de Costa Rica, San Jose, Costa Rica

Everhart, S., Oregon State University, Corvallis, OR, U.S.A.

Everts, K. L., University of Maryland/University of Delaware, Salisbury, MD, U.S.A.

Fernando, D. G., University of Manitoba, Winnipeg, Manitoba, Canada

Faedda, R., University of Catania, Catania, Italy

Farman, M., University of Kentucky, Lexington, KY, U.S.A.

Fellers, J., USDA-ARS, Manhattan, KS, U.S.A.

Fetch, T., Cereal Research Centre, AAFC, Winnipeg, Manitoba, Canada

Figueroa, M., University of Minnesota, St. Paul, MN, U.S.A.

Flors, V., Castellón, Spain

Foissac, X., INRA, Université de Bordeaux, Villenave d'Ornon, France

Forbes, G., Lima, Peru

Fraaije, B., Rothamsted Research, BCCP, Hertfordshire, United Kingdom

Fraile, A., Universidad Politécnica de Madrid, Madrid, Spain Friskop, A., North Dakota State University, Fargo, ND, U.S.A.

Fuchs, M., Cornell University, Geneva, NY, U.S.A.

Gao, Q., University of Kentucky, Lexington, KY, U.S.A.

Gadoury, D., Cornell University, NYSAES, NY, U.S.A.

Gal-On, A., Plant Protection, Plant Pathology, Bet-Dagan, Israel

Gamliel, A., Agricultural Engineering, Pest Management, Bet-Dagan, Israel

Garbelotto, M., University of California, ESPM, Berkeley, CA, U.S.A.

Garcia-Arenal, F., Universidad Politecnica de Madrid, Madrid, Spain

Gartemann, K.-H., University of Bielefeld, Bielefeld, Germany

Gautum, P., North Dakota State University, Langdon, ND, U.S.A.

Gebhardt, C., Max Planck Institute for Plant Breeding Research

Geiser, D., Penn State University, University Park, PA, U.S.A.

Gelvin, S. B., Purdue University, West Lafayette, IN, U.S.A.

Gent, D. H., USDA-ARS, Corvallis, OR, U.S.A.

Gentry, T., College Station, TX, U.S.A.

Gevens, A. J., University of Wisconsin, Madison, WI, U.S.A.

Giblin, R. D., University of Florida-IFAS, Davie, FL, U.S.A.

Gidoin, C., Supagro, Montpellier, France

Giraldo, M. C., Kansas State University, Manhattan, KS, U.S.A.

Glasa, M., Institute of Virology, Slovak Academy of Sciences,

Bratislava, Slovakia

Gonzalez, C., Texas A\&M University, College Station, TX, U.S.A.

Goodwin, S., USDA-ARS/Purdue University, West Lafayette, IN, U.S.A.

Gordon, T., University of California, Davis, CA, U.S.A.

Goss, E. M., University of Florida, Gainesville, FL, U.S.A.

Gottula, J. W., Cornell University, Geneva, NY, U.S.A.

Govindarajulu, M.

Grant, M., Exeter, United Kingdom

Grenville-Briggs, L., Swedish University of Agricultural Sciences,

Alnarp, Sweden

Grisham, M. P., USDA, ARS, SRRC, Houma, LA, U.S.A.

Grünwald, N. J., USDA-ARS, Corvallis, OR, U.S.A.

Gugino, B. K., The Pennsylvania State University, University Park, PA, U.S.A.

Guo, L., China Agricultural University, Beijing, China

Guttman, D. S., University of Toronto, Ontario, Canada

Hahn, M., Kaiserslautern University, Kaiserslautern, Germany

Halterman, D., USDA-ARS, Madison, WI, U.S.A.

Ham, J. H., Rothamsted Research, Harpenden, United Kingdom

Hammerschmidt, R., Michigan State University, East Lansing, MI,

U.S.A.

Hammond, J., USDA-ARS, Beltsville, MD, U.S.A.
Hammond, R., USDA-ARS, Beltsville, MD, U.S.A.

Hanke, M.-V., Julius Kühn-Institut, Germany

Hanson, L., USDA-ARS, East Lansing, MI, U.S.A.

Harrison, N., University of Florida, Fort Lauderdale, FL, U.S.A.

Hart, J., Cornell University, Ithaca, NY, U.S.A.

Hartman, G., USDA-ARS \& UI, Urbana, IL, U.S.A.

Harveson, R., University of Nebraska, Scottsbluff, NE, U.S.A.

He, Y. W., Shanghai Jiao Tong University, Shanghai, China

He, Y., North Carolina State University, Raleigh, NC, U.S.A.

Hebrard, E., IRD, RPB, Montpellier, France

Henry, B., Mississippi State, MS, U.S.A.

Heuer, H., Julius Kühn-Institut, Münster, Germany

Hietala, A. M., Norwegian Forest and Landscape Institut, Aas, Norway

Hillman, B., Rutgers University, New Brunswick, NJ, U.S.A.

Holland, J., USDA-ARS, Raleigh, NC, U.S.A.

Holmes, G. J., California Polytechnic State University, San Luis Obispo, CA, U.S.A.

Hong, C., Virginia Polytechnic Institute and State University, Virginia Beach, VA, U.S.A.

Horwitz, B.

Huber, D. M., Purdue University, West Lafayette, IN, U.S.A.

Hughes, T., Monsanto, Chesterfield, MO, U.S.A.

Hwang, I.

Höfte, M., Ghent University, Gent, Belgium

Inderbitzin, P., University of California, Davis, CA, U.S.A.

Irey, M., U.S.A. Sugar Corporation/Southern Gardens Citrus, Clewiston, FL, U.S.A.

Isard, S., Pennsylvania State University, University Park, PA, U.S.A.

Ishiguro, K., MAFF/AFFRC, Tsukuba, Ibaraki, Japan

Ishimaru, C., University of Minnesota, St. Paul, MN, U.S.A.

Ivors, K., California Polytechnic State University, San Luis Obispo, CA, U.S.A.

Iyer-Pascuzzi, A., Purdue University, West Lafayette, IN, U.S.A.

Jackson, R., University of Reading, Reading, United Kingdom

Jacobsen, B., Montana State University, Bozeman, MT, U.S.A.

Jacques, M.-A., UMR PaVé, Beaucouzé Cedex, France

Jafra, S., University of Gdansk, Gdansk, Poland

Jamann, T., Cornell University, Ithaca, NY, U.S.A.

James, D., Sidney Laboratory, Canadian Food Inspection Agency, Sidney, British Columbia, Canada

Jardine, D., Kansas State University, Manhattan, KS, U.S.A.

Jeger, M. J., Imperial College London, Ashford, United Kingdom

Ji, P., University of Georgia, Athens, GA, U.S.A.

Jiang, C.-J., National Institute of Agrobiological Sciences, Tsukuba, Japan

Jiménez-Díaz, R. M., Instituto Agricultura Sostenible, Córdoba, Spain

Jiménez-Gasco, M., Pennsylvania State University, University Park, PA, U.S.A.

Johnson, D. A., Washington State University, Pullman, WA, U.S.A.

Johnson, E. G., University of Florida, Lake Alfred, FL, U.S.A.

Johnson, K., Department of Botany and Plant Pathology, Corvallis, OR, U.S.A.

Jones, J., The James Hutton Institute, Dundee, United Kingdom

Jones, J. B., University of Florida, Gainesville, FL, U.S.A.

Jones, R., USDA-ARS, Beltsville, MD, U.S.A.

Jordan, K., University of Guelph, Guelph, Cameroon

Jordan, R., U.S. National Arboretum, USDA-ARS, Beltsville, MD, U.S.A.

Jurick, W. M., II, USDA-ARS, Beltsville, MD, U.S.A.

Juvale, P., Iowa State University, Ames, IA, U.S.A.

Juzwik, J., USDA Forest Service, St. Paul, MN, U.S.A.

Kabbage, M., University of Wisconsin, Madison, WI, U.S.A.

Kangasjarvi, S.

Karasev, A., University of Idaho, Moscow, ID, U.S.A.

Kasson, M. T., Virginia Tech, Blacksburg, VA, U.S.A.

Keizerweerd, A., USDA, Houma, LA, U.S.A.

Kelly, J., Michigan State University, East Lansing, MI, U.S.A.

Kennedy, G. G., North Carolina State University, Raleigh, NC, U.S.A. 
Kerns, J., North Carolina State University, Raleigh, NC, U.S.A. Khan, M. F., North Dakota State University \& University of Minnesota, Fargo, ND, U.S.A.

Kiewnick, S., Agroscope Changins-Waedenswil, Waedenswil, Switzerland

Killiny, N., University of Florida, IFAS, Lake Alfred, FL, U.S.A.

Kim, Y.-K. (Richard), Pace International, Wapato, WA, U.S.A.

Kistler, H. C., USDA-ARS, St. Paul, MN, U.S.A.

Kitajima, E., Universidade de Sao Paulo, Escola Superior de Agricultura Luiz de Queiroz, Piracicaba, Sao Paulo, Brazil

Klosterman, S. J., USDA-ARS, Salinas, CA, U.S.A.

Koike, S. T., Cooperative Extension Monterey County, Salinas, CA, U.S.A.

Kolkman, J., Cornell University, Ithaca, NY, U.S.A.

Kolmer, J., USDA-ARS, St. Paul, U.S.A.

Kolomiets, M. V., Texas A\&M University, College Station, TX, U.S.A.

Krenz, B., Cornell University, Ithaca, NY, U.S.A.

Kuhn, H., RWTH Aachen University, Aachen, Germany

Lemaire, O. J.-M., INRA, Colmar, Elsass, France

Lai, E.-M., Academia Sinica, Taipei, Taiwan

Lambert, K., University of Illinois, Urbana, IL, U.S.A.

Lamour, K., University of Tennessee, Knoxville, TN, U.S.A.

Lang, J., Colorado State University, Fort Collins, CO, U.S.A.

Larajeira, F., Embrapa Cassava \& Fruits, Cruz das Almas, Bahia, Brazil

Le Clerc, V., Agrocampus Ouest-INHP, Angers, France

LeBoldus, J. M., North Dakota State University, Fargo, ND, U.S.A.

Lee, G. P., Chung-Ang University, Aunsung, Republic of Korea

Lee, R. F., USDA-ARS, NCGRCD, Riverside, CA, U.S.A.

Lehman, J. S., Otterbein College, Westerville, OH, U.S.A.

Lewis, E., University of California, Davis, CA, U.S.A.

Li, W., USDA APHIS, CPHST, Beltsville, MD, U.S.A.

Liao, Y.-C., Huazhong Agricultural University, Wuhan, HuBei, China

Lin, H., USDA-ARS, CDPG, Parlier, CA, U.S.A.

Ling, K.-S., USDA-ARS, Charleston, SC, U.S.A.

Little, C. R., Kansas State University, Manhattan, KS, U.S.A.

Lu, S., Mississippi State University, MS, U.S.A.

Luo, W., USDA-ARS, Fort Pierce, FL, U.S.A.

MORI, N., University of Padova, Legnaro, Padova, Italy

Ma, Z., Plant Pathology, Hangzhou, China

Madden, L. V., Ohio State University, Wooster, OH, U.S.A.

Magill, C., Texas A\&M University, College Station, TX, U.S.A.

Mahaffee, W., USDA-ARS, Corvallis, OR, U.S.A.

Makowski, D., INRA, Thiverval-Grignon, France

Malbran, I., Universidad Nacional de La Plata, Buenos Aires, Argentina

Malvick, D., University of Minnesota, St. Paul, MN, U.S.A.

Marchant, B.

Maree, $\mathrm{H}$.

Marine, S., University of Maryland, Salisbury, MD, U.S.A.

Marois, J. J., University of Florida, Quicny, FL, U.S.A.

Martin, B., Clemson University, Florence, SC, U.S.A.

Martin, F. N., USDA-ARS, Salinas, CA, U.S.A.

Marzachí, C., CNR, Istituto di Virologia Vegetale, Torino, Italy

Mavrodi, D., Washington State University, Pullman, WA, U.S.A.

Mazzola, M., USDA-ARS, Wenatchee, WA, U.S.A.

McCallum, B., Agriculture and Agri-Food Canada, Winnipeg, Manitoba, Canada

McCann, H., Massey University, New Zealand Institute for Advanced Study, Auckland, New Zealand

McCartney, C., Agriculture Agri-Food Canada, Morden, Canada,

McCormick, S., USDA-ARS-NCAUR, Peoria, IL, U.S.A.

McGrath, M., Cornell University, Riverhead, NY, U.S.A.

McManus, P. S., University of Wisconsin, Madison, WI, U.S.A.

McRoberts, N., University of California, Davis, CA, U.S.A.

McSpadden Gardener, B. B., Ohio State University-OARDC,

Wooster, OH, U.S.A.

Mead, A., Rothamsted Research, Harpenden, United Kingdom
Meinhardt, S., North Dakota State University, Fargo, ND, U.S.A. Melcher, U., Oklahoma State University, Stillwater, OK, U.S.A. Melzer, M. J., University of Hawaii, Honolulu, HI, U.S.A.

Meyer, S., USDA-ARS, Beltsville, MD, U.S.A.

Mideros, S. X., Cornell University, Ithaca, NY, U.S.A.

Mila, A., North Carolina State University, Raleigh, NC, U.S.A.

Miles, T. D., Michigan State University, East Lansing, MI, U.S.A.

Milijašević-Marčić, S., Institute of Pesticides and Environmental

Protection, Belgrade-Zemun, Serbia

Miller, L., University of Missouri, Columbia, MO, U.S.A.

Miller, W., USDA-ARS, Albany, CA, U.S.A.

Milus, G., University of Arkansas, Fayetteville, AR, U.S.A.

Miranda, L., Bayer Crop Science, Pikeville, NC, U.S.A.

Mitchell, T., The Ohio State University, Columbus, OH, U.S.A.

Mitchell, T. R., USDA-ARS, Athens, GA, U.S.A.

Mitter, N., The University of Queensland, QAAFI, Brisbane,

Queensland, Australia

Mizubuti, E., Universidade Federal de Vicosa, Vicosa, MG, Brazil

Montarry, J., INRA, UMR INRA, Le Rheu, France

Moretti, A., CNR, ISPA, Bari, Italy

Moury, B., INRA, Montfavet, France

Mueller, D., Iowa State University, Ames, IA, U.S.A.

Mugnai, L., University of Florence, Florence, Italy

Mundt, C., Oregon State University, Corvallis, OR, U.S.A.

Munkvold, G., Iowa State University, Ames, IA, U.S.A.

Munyaneza, J., USDA-ARS, WA, U.S.A.

Muthomi, J.

Nagata, T., Universidade de Brasília, Brasília, DF, Brazil

Navas-Cortés, J. A., Cordoba, Spain

Nelson, C. D., USDA Forest Service, Saucier, MS, U.S.A.

Nicot, P., INRA, Montfavet, France

Niks, R. E., Wageningen University, Wageningen, Netherlands

Nita, M., Virginia Tech, Winchester, VA, U.S.A.

Nunney, L., University of California, Riverside, CA, U.S.A.

Nykyri, J.

O'Donnell, K., USDA, ARS, NCAUR, MGB, Peoria, IL, U.S.A.

Ocamb, C. M., Oregon State University, Corvallis, OR, U.S.A.

Olaya, G., Syngenta Crop Protection, Vero Beach, FL, U.S.A.

Oldenburg, D.

Oliver, J., Kansas State University, Manhattan, KS, U.S.A.

Oliver, R. P., Murdoch University, Perth, Western Australia, Australia

Olivier, C., Agriculture and Agri-Food Canada, Saskatoon,

Saskatchewan, Canada

Olukolu, B., North Carolina State University, Raleigh, NC, U.S.A.

Oster, J., California Cooperative Rice Research Foundation, Inc.,

Biggs, CA, U.S.A.

Oudemans, P. V., Rutgers University, Chatsworth, NJ, U.S.A.

Pruvost, O., CIRAD, Saint Pierre, Reunion, France

Pallás Benet, V., IBMCP (CSIC-UPV), Valencia, Spain

Palumbo, J. D., USDA-ARS, Albany, CA, U.S.A.

Panabières, F., INRA, Institut Sophia Agrobiotech, Sophia Antipolis, France

Panthee, D., North Carolina State University, Mills River, NC, U.S.A.

Paran, I., Institute of Plant Sciences, Volcani Center, Israel

Parke, J., Oregon State, Corvallis, OR, U.S.A.

Parnell, S., Rothamsted Research, Harpenden, Hertfordshire, United Kingdom

Paul, P. A., Ohio State University, Wooster, OH, U.S.A.

Paulitz, T. C., USDA-ARS, Pullman, WA, U.S.A.

Periyannan, S., CSIRO, Australian Capital Territory, Australia

Perry, K. L., Cornell University, Ithaca, NY, U.S.A.

Peterschmitt, M., CIRAD, BGPI, Montpellier Cedex 5, France

Pierson, E., Texas A\&M University, College Station, TX, U.S.A.

Ploetz, R., University of Florida, Homestead, FL, U.S.A.

Poland, J., USDA-ARS, Manhattan, KS, U.S.A.

Pretorius, Z., University of the Free State, Bloemfontein, South Africa

Price, J. A., Texas Agrilife, Bushland, TX, U.S.A.

Ptacek, J.

Qian, G., Nanjing, Jiangsu, China 
Qu, F., Ohio State University, Wooster, OH, U.S.A.

Quesada-Ocampo, L. M., East Lansing, U.S.A.

Rahman, H., University of Alberta, Edmonton, Alberta, Canada

Raid, R. N., University of Florida, IFAS, Belle Glade, FL, U.S.A.

Ramirez, C., University of Antioquia, Medellin, Antioquia, Colombia

Rampitsch, C., Agriculture \& Agri-Food Canada, Winnipeg,

Manitoba, Canada

Rashed, A., University of Idaho, PSES, Aberdeen, ID, U.S.A.

Ravelonandro, M., INRA-Bordeaux, Villenave d'Ornon, France

Regoes, R., ETH Zurich, Switzerland

Reignault, P. L., Université du Littoral Côte d'Opale UCEIV, Calais, France

Renton, M., CSIRO Ecosystems Sciences, Perth, Western Australia, Australia

Ritchie, D., North Carolina State University, Raleigh, U.S.A.

Roberts, P., University of Florida, Immokalee, FL, U.S.A.

Roberts, P. A., University of California, Riverside, CA, U.S.A.

Rosa, C., Pennsylvania State University, University Park, PA, U.S.A.

Rossi, V., Università Cattolica del Sacro Cuore, Piacenza, Italy

Rotenberg, D., Kansas State University, Manhattan, KS, U.S.A.

Rouse, D. I., University of Wisconsin, Madison, WI, U.S.A.

Rouse, M. N., USDA-ARS, St. Paul, MN, U.S.A.

Rowhani, A., University of California, Davis, CA, U.S.A.

Rubio, L., Instituto Valenciano de Investigaciones Agrarias,

Moncada, Valencia, Spain

Rudd, J., Rothamsted Research

Rupe, J. C., University of Arkansas, Fayetteville, AR, U.S.A.

Saldarelli, P., CNR-Institute for Sustainable Plant Protection, CNRAgroalimentare, Bari, Italy

San Jose Garcia, M., University of Reading, Berkshire, United Kingdom

Sanogo, S., New Mexico State University, EPPWS, Las Cruces, NM, U.S.A.

Savary, S., INRA, UMR AGIR, Castanet Tolosan, France

Sawa, S.

Schena, L., Mediterranean University of Reggio Calabria, Reggio Calabria, Italy

Scherm, H., University of Georgia, Athens, GA, U.S.A.

Schilder, A., Michigan State University, East Lansing, MI, U.S.A.

Schmale, D., Virginia Polytechnic Institute and State University,

Blacksburg, VA, U.S.A.

Schnabel, G., Clemson University, Clemson, SC, U.S.A.

Scholthof, H., College Station, U.S.A.

Schwartz, H. F., Colorado State University, Fort Collins, CO, U.S.A.

Scortichini, M., CRA-Istituto Sperimentale per la Frutticoltura, Biology, Roma, Italy

Scott, J., University of Tasmania, Burnie, Tasmania, Australia

Scott, S., Clemson University, SAFES, Clemson, SC, U.S.A.

Sederoff, R., North Carolina State University, Raleigh, U.S.A.

Seebold, K., Wesley, University of Kentucky, Lexington, KY, U.S.A.

Shaw, M., University of Reading, Reading, United Kingdom

Shim, W.-B., Texas A\&M University, College Station, TX, U.S.A.

Short, D. P. G., University of California, Davis, Salinas, CA, U.S.A.

Sierotzki, H., Syngenta Crop Protection, Research Biology, Stein,

Switzerland

Simko, I., USDA-ARS, Salinas, CA, U.S.A.

Singh, J., Agriculture and Agri-Food Canada, Ottawa, Ontario Canada

Sipes, B., University of Hawaii, Honolulu, HI, U.S.A.

Skelsey, P., The James Hutton Institute, Dundee, United Kingdom

Smart, C. D., Cornell University, NYSAES, Geneva, NY, U.S.A.

Smith, K. P., University of Minnesota, St. Paul, MN, U.S.A.

Somerville, S., University of California, Berkeley, CA, U.S.A.

Srinivasan, R., University of Georgia, Tifton, GA, U.S.A.

St. Clair, D., University of California-Davis, CA, U.S.A.

Steffenson, B. J., University of Minnesota, St. Paul, MN, U.S.A.

Stenger, D. C., USDA-ARS, Parlier, CA, U.S.A.

Stenglein, S., Azul, Argentina

Stewart, J. E., USDA-ARS, Corvallis, OR, U.S.A.

Stewart, L. R., USDA-ARS, Wooster, OH, U.S.A.
Stockwell, V., Oregon State University, Corvallis, OR, U.S.A.

Strehlow, B., University of Rostock, Rostock, Germany

Sudarshana, M., USDA-ARS, Davis, CA, U.S.A.

Sugar, D., Oregon State University, Medford, OR, U.S.A.

Sundin, G. W., Michigan State University, East Lansing, MI, U.S.A.

Sutherland, M. W., University of Southern Queensland, Toowoomba, Queensland, Australia

Suzuki, J., USDA-ARS, Hilo, HI, U.S.A.

Swett, C., University of Maryland, College Park, MD, U.S.A.

Szabo, L., USDA-ARS, St. Paul, MN, U.S.A.

Sándor, E., University of Debrecen, Debrecen, Hungary

Takahashi, F., Shizuoka Prefectural Research Institute of Agriculture and Forestry, Kikugawa, Shizuoka, Japan

Tan, K.-C., Perth, Western Australia, Australia

Terauchi, R., Iwate Biotechnology Research Center, Kitakami, Iwate, Japan

Tharreau, D., CIRAD, UMR BGPI, Montpellier, France

Thomas, C. S., University of California, Davis, CA, U.S.A.

Thompson, J. R., Cornell University, Ithaca, NY, U.S.A.

Thébaud, G., INRA, UMR BGPI, Montpellier, France

Timmer, P., Citrus Research and Education Center, Lake Alfred, FL, U.S.A.

Tjamos, E., Agricultural University Athens, Athens, Greece

Tomaso-Peterson, M., Mississippi State University, Mississippi State, MS, U.S.A.

Toussaint, V., Agriculture and Agri-Food Canada, St-Jean-surRichlieu, Quebec, Canada

Triplett, L., Colorado State University, Fort Collins, CO, U.S.A.

Tsai, C.-W., National Taiwan University, Taipei, Taiwan

Turechek, W. W., USDA-ARS USHRL, Fort Pierce, FL, U.S.A.

Tylka, G., Iowa State University, Ames, IA, U.S.A.

Typas, M., University of Athens, Athens, Greece

Uphaus, J., DuPont Pioneer, Windfall, IN, U.S.A.

Valent, B., Kansas State University, Manhattan, KS, U.S.A.

van Alfen, N., University of California-Davis, CA, U.S.A.

van Bruggen, A. H. C., University of Florida, Gainesville, FL, U.S.A.

Verdier, V., Institut de Recherche pour le Développement,

Montpellier, France

Vogel, J.

Walker, A.-S., INRA, Bioger-CPP, Thiverval Grignon, France

Walker, N. R., Oklahoma State University, Stillwater, OK, U.S.A.

Wallis, C., USDA-ARS, Parlier, CA, U.S.A.

Walters, D., SRUC, Edinburgh, United Kingdom

Wang, D., Los Alamos, U.S.A.

Wang, G.-F., North Carolina State University, Raleigh, NC, U.S.A.

Wang, N., University of Florida, Lake Alfred, FL, U.S.A.

Wang, X., USDA-ARS, Ithaca, NY, U.S.A.

Wanner, L. A., USDA-ARS, Beltsville, MD, U.S.A.

Ward, T., USDA-ARS, Peoria, IL, U.S.A.

Wayadande, A. C., Oklahoma State University, Stillwater, OK, U.S.A.

Webb, S., University of Florida, Gainesville, FL, U.S.A.

Webster, C.

Webster, D., Rothamsted Research, Harpenden, United Kingdom

Wechter, W. P., USDA-ARS, Charleston, SC, U.S.A.

Wegulo, S., University of Nebraska, Lincoln, NE, U.S.A.

Weller, D.

Wellings, C., University of Sydney, New South Wales, Australia

Werres, S., Julius Kuehn Institut (JKI), Braunschweig, Germany

West, J., Rothamsted Research, Harpenden, United Kingdom

Westphal, A., Julius Kühn-Institut, Münster, Germany

Wheeler, T., Texas AgriLife Research, Lubbock, TX, U.S.A.

Whitfield, A., Kansas State University, Manhattan, KS, U.S.A.

Wick, R., University of Massachusetts, Amherst, MA, U.S.A.

Wilcox, W. F., Cornell University, Geneva, NY, U.S.A.

Workneh, F., Texas Agricultural Experiment Station, Bushland, TX, U.S.A.

Wright, R., Texas Tech University, Lubbock, TX, U.S.A.

Wu, B., China Agricultural University, Beijing, China

Wubben, M., USDA-ARS, Mississippi State, MS, U.S.A.

Wyenandt, C. A., Rutgers University, East Brunswick, NJ, U.S.A. 
Xiao, C.-L., Washington State University, Wenatchee, WA, U.S.A.

$\mathrm{Xu}, \mathrm{X}$., East Malling Research, West Malling, Kent, United Kingdom Yeh, S.-D., National Chung Hsing University, Taichung City, Taiwan Yepes, M., Cornell University, Geneva, NY, U.S.A.

Yin, C., Washington State University, Pullman, WA, U.S.A.

Yuen, J. E., Swedish University of Agricultural Science, Uppsala, Sweden
Zeng, L., University of Arkansas, Little Rock, AR, U.S.A.

Zeng, Q., Michigan State University, East Lansing, MI, U.S.A.

Zerbini, M., Universidade Federal de Vicosa, Minas Gerais, Brazil

Zhang, Y., Institute of Microbiology, Beijing, China

Zhong, S., North Dakota State University, Fargo, ND, U.S.A.

Zhou, S., Texas A\&M University, Beaumont, TX, U.S.A.

Ziebell, H., Julius Kuehn-Institut, Braunschweig, Germany 